\title{
TỔNG QUAN MộT SỐ NGHIÊN CỨU CHI PHÍ HIỆU QUẢ LIỆU PHÁP ĐIỀU TRI ĐÍCH UNG THƯ PHỔI KHÔNG TẾ BÀO NHỎ (UTPKTBN)
}

\author{
Nguyễn Văn Chỉnh', Phạm Cẩm Anh², Phạm Huy Tuấn Kiệt ${ }^{2}$ \\ Trần Thị Thanh Hương ${ }^{3}$, Nguyễn Thị Thái Hòa ${ }^{3}$
}

\section{TÓM TẮT}

Mục tiêu: Liệu pháp nhắm trúng đích trong điều tri UTPKTBN sẽ kéo dài thời gian sông của người bênh hớn so với hóa trị liệu nhưng chi phí (CP) lại khá cao. Do vây, trong nghiền cứu này, chúng tôi sẽ tìm ra bằng chứng về chi phí hiệu qưa (CPHQ) của các liệu pháp nhắm trúng đích so với hóa trị liẹu nhằm đưa ra khuyến nghị trong việc lựa chọn phương pháp điều trị phù hợp. Phương pháp nghiên cứu: Tiến hành tổng quan tài liệu một các có hệ thống để đánh giá $C P H Q$ của liệu pháp nhắm trúng đích trong điêu trị UTPKTBN dựa trên các cơ sở dữ liệu Pubmed, Embase, Cochrane,.. từ năm 2000 đến tháng 8 năm 2021. Đánh giá chất lượng của các nghiên cứu Kinh tế Y tế bằng bảng kiểm CHEERS. Kết quả: Sáu trong 128 kết quả đã đáp ứng đầy đủ các tiêu chí theo bảng kiểm CHEERS và được đưa vào nghiên cứu. ICER của erlotinib, afatinib và osimertinib lânn lượt là $85.927,4$ \$/QALY, 20.758-33.416,39\$/QALY và 23.760,1$51.615,1 \$ / Q A L Y$ so với các liêu pháp hóa tri liệu. Kết luận: Erlotinib và afatinib là phác đồ bước một và osimertinib là phác đồ bước sau có $\mathrm{CPHQ}$ cao hơn so với hóa trị. Giảm giá thuốc điêuu trị nhắm trúng đích thấp hơn so với ngưỡng chi trả của từng nước là yếu tố thuận lợi để người bệnh UTPKTBN có khả năng tiếp cân và gia tăng $\mathrm{CPHQ}$.

Tư khóa: Ung thư phổi không tế bào nhỏ, chi phí hiệu quả, điều trị nhắm trúng đích.

\section{SUMMARY}

A LITERATURE REVIEW OF THE COSTEFFECTIVENESS OF TARGETED THERAPIES IN THE TREATMENT OF NON-SMALL CELL LUNG CANCER (NSCLC)

Objects: Targeted therapy in the treatment of non-small cell lung cancer will prolong the patient's life longer than chemotherapy option, but its cost is higher. Therefore, in this study, we will find evidences on the cost-effectiveness of targeted therapy compared with chemotherapy method to provide recommendations in choosing the appropriate treatment. Methods: Conducting a systematic literature review on cost-effectiveness, QALYs,... based on Pubmed, Embase and Cochrane database,...

${ }^{1}$ Trung tâm xét nghiệm Dr.LABO, Công ty cổ phần chân đoán Y hoc Hà Nội.

2Trường Đại học Y Hà Nội.

${ }^{3}$ Viện Ung thu Quốc gia.

Chịu trách nhiệm chính: Nguyễn Văn Chỉnh

Email: giaosuchinh@gmail.com

Ngày nhận bài: 23.8.2021

Ngày phản biên khoa họ: 18.10.2021

Ngày duyệt bài: 28.10 .2021 from 2000 to August 2021. Studies used the CHEERS checklist. Results: Six out of 128 results having the full criterions were suitable for the CHEERS checklist and were included in the study. The ICERs of erlotinib, afatinib and osimertinib were \$85927.4/QALY, \$20758-33416.39/QALY, and \$23760.1$51615.1 / \mathrm{QALY}$ respectively compared to other chemotherapy treatment options. Conclusion: Erlotinib and afatinib for first-line treatment and osimertinib for second-line treatment options are more cost-effective than chemotherapy methods. Reducing the price of targeted drugs lower than the payment threshold of each country will play a vital value for non-small cell lung cancer patients to have access and the economic outcome could become more favorable.

Key word: Non-small cell lung cancer, cost effectiveness, targeted therapy method.

\section{I. ĐẶT VẤN ĐỀ}

Chi phí (CP) điều trị ung thư phổi (UTP) đã gia tăng gấp đôi tại Mỹ (2020) [1-3]. UTPKTBN chiếm $85-90 \%$ các trường hợp UTP [3-6], với khoảng $80 \%$ ở giai đoạn tiên triển (giai đoạn IIIb/IV) khi được chẩn đoán [3]. Có khoảng 10$15 \%$ bệnh nhân Châu Âu và $50 \%$ bệnh nhân châu Á có đôt biến EGFR dương tính [5-6]. Phác đồ điêu trị UTPKTBN bằng hóa trị liệu toàn thân dựa trên plastin (cisplatin hoặc carboplatin) kết hợp với pemetrexed và gemcitabine thường kém hiệu quả và thời gian sống sót trung bình chỉ gân 1 năm [4]. Các chất ức chế EGFR-tyrosine kinase (TKIs) thuộc thế hệ thứ nhất (gefitinib, erlotinib) và thứ hai (afatinib, dacomitinib), thứ ba (osimertinib) là những phương pháp điêu trị nhắm trúng đích có tỷ lệ đáp ứng, thời gian sống thêm không tiến triển cao hơn và tỷ lệ tác dụng phụ nghiêm trọng thấp hơn so với hóa trị liệu dựa trên plastin trong quân thể có đột biến EGFR [4].

Tuy nhiên, CP điều trị bằng TKIs khá cao là rào cản để bệnh nhân tiếp cận được thuốc. Do vậy, chúng tôi sẽ tổng hợp có hệ thống các bằng chứng kinh tế dược như: CPHQ, ICER, QALY,... của phương pháp điều trị nhắm trúng đích trong điêu trị UTPKTBN có EGFR dương tính ở giai đoạn IIIb, IV so với các phương pháp hóa tri. Chất lượng của các nghiên cứu được đánh giá thông qua một công cụ đánh giá chất lượng kinh tế y tế (CHEERS) [7].

\section{PHƯƠNG PHÁP NGHIÊN CỨU \\ Câu hỏi nghiên cứu: Xây dựng dựa trên}


khung PICOS (Đối tượng - Can thiệp - So sánh Kết quả - Thiết kế nghiên cứu) nhằm xác định mục tiêu của nghiên cứu.

Chiến lược tìm kiếm tài liệu: Kết hợp các từ khóa và toán tử OR hoặc AND: ((cancer OR lung cancer OR non small lung cancer OR non small cell bronchial carcinoma OR NSCLC) AND (stage IIIb AND stage IV) AND (cost-effectiveness OR cost effect/cost-effective OR cost efficient OR beneficial cost) AND targeted therapy).

Tiêu chuẩn lựa chọn và loại trừ: Các nghiên cứu được lựa chọn dựa trên các tiêu chuẩn lựa chọn và loại trừ (Hình 1).
Tổng hợp và xử lý thông tin: Hai nghiên cứu viên độc lập sẽ tiến hành tìm kiếm, tổng hợp dựa trên Bảng 1 và Bảng 2: Tác giả, quốc gia, CPHQ, QALY, ICER,.... Các kết quả thu được sẽ được đối chiếu nếu có bất thường sẽ thảo luận nhóm để đưa ra kết luân. Các loại CP sẽ được đổi sang đô la Mỹ (\$) trong năm quy đổi đề thuận tiện cho việc so sánh [8].

Đánh giá chất lượng nghiên cứu: Các nghiên cứu sẽ được đánh giá chất lượng bằng bảng kiểm CHEERS [7]. Các nghiên cứu được đánh giá là đạt chất lượng khi kết quả tổng hợp 24 tiêu chí có tổng số điểm >19/24 điểm sẽ được đưa vào nghiên cứu.

\section{Tiêu chuẩn lựa chọn:}

1.Đối tượng nghiên cứu là người bệnh UTPKTBN, giai đoạn IIIb, IV và có xét nghiệm EGFR dương tính.

2. Ngôn ngữ: Tiếng Anh.

3. Nghiên cứu công bố: Bản đây đủ và có CPHQ, ICER, QALY,..

4. Thời gian cống bố: Từ năm 2000 tới tháng 8 năm 2021.

\section{Tiêu chuẩn loại trừ:}

1. Người bệnh là ung thư phổi tế bào nhỏ hoặc giai đoạn I, II, IIIa hoặc có xét nghiệm EGFR âm tính. 2. Ngôn ngữ: Không phải tiếng Anh. 3. Nghiên cứu công bố: Bản tóm tắt hoặc bản sao hoặc chỉ có chi phí đơn thuần.

4. Thời gian công bố: Trước năm 2000.

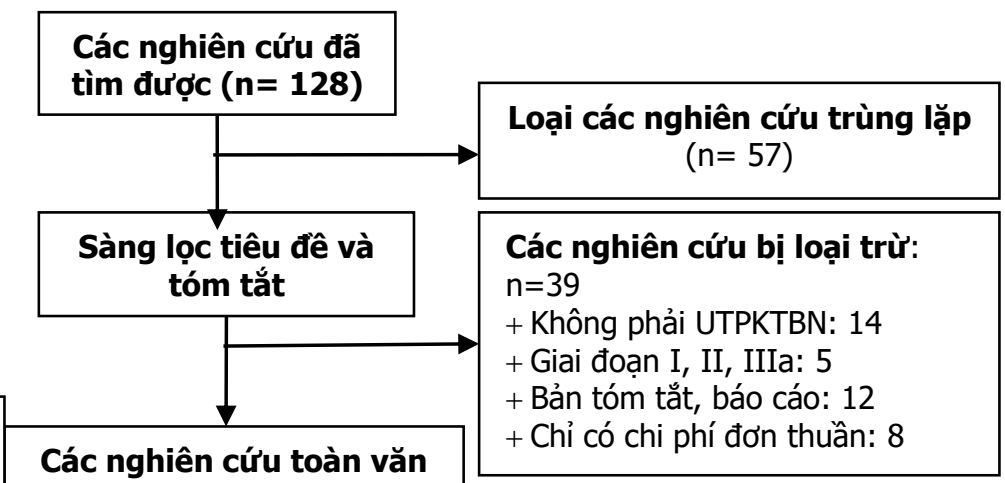

và đủ điều kiện lựa chọn $(n=13)$

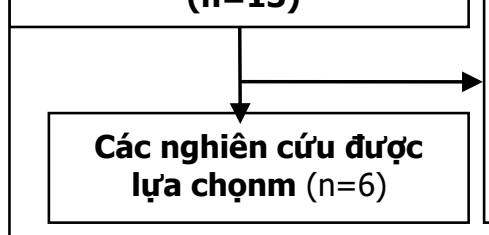

Nghiên cứu đây đủ bị loại trừ: $n=7$

+ Không phải tiếng Anh: 2

+ Chỉ có chi phí đớn thuân: 1

+ Bản nhận xét: 1

+ Không liên quan: 3

\section{KẾT QUẢ NGHIÊN CứU}

Hình 1. Quá trinh tìm kiếm và lựa chọn kết quả nghiên cứu

3.1. Đánh giá chất lượng các kết quả nghiên cứu (CHEERS)

Bảng 1. Kết quả đánh giá chất lượng các nghiên cứu (CHEERS)

\begin{tabular}{|c|c|c|c|c|c|c|c|c|}
\hline Mục & Nội dung & $\begin{array}{c}\text { Mứ } \\
\text { số }\end{array}$ & $\begin{array}{c}\text { Alain } \\
{[1]}\end{array}$ & $\begin{array}{c}\text { Siying } \\
{[2]}\end{array}$ & $\begin{array}{c}\text { Xiaohua } \\
{[3]}\end{array}$ & $\begin{array}{c}\text { Ruxu } \\
{[4]}\end{array}$ & $\begin{array}{c}\text { Evelina } \\
{[5]}\end{array}$ & $\begin{array}{c}\text { Haijing } \\
{[6]}\end{array}$ \\
\hline \multicolumn{9}{|c|}{ Tiêu đề và tóm tăt } \\
\hline Tiêu đề & $\begin{array}{l}\text { Thể hiện nghiên cứu đánh } \\
\text { giá kinh tế }\end{array}$ & 1 & 1 & 1 & 1 & 1 & 1 & 1 \\
\hline Tóm tắt & $\begin{array}{l}\text { Đầy đủ: mứ tiêu, quan điểm, } \\
\text { phương pháp, kết quả, kêt luận }\end{array}$ & 2 & 1 & 1 & 1 & 1 & 1 & 1 \\
\hline \multicolumn{9}{|c|}{ Đặt vấn đề } \\
\hline $\begin{array}{l}\text { Giới thiêu, } \\
\text { mục tiếu }\end{array}$ & $\begin{array}{l}\text { Nêu hoàn cảnh, câu hỏi } \\
\text { nghiên cứu rố ràng }\end{array}$ & 3 & 1 & 1 & 1 & 1 & 1 & 1 \\
\hline \multicolumn{9}{|c|}{ Phương pháp } \\
\hline Quần thể & $\begin{array}{l}\text { Miêu tả các đăă } \\
\text { lựa chọn c }\end{array}$ & 4 & 1 & 1 & 1 & 1 & 1 & 1 \\
\hline Cẫu trúc & $\begin{array}{l}\text { Mô tá các mặt cúa vân đề từ } \\
\text { đó đưa ra được lựa chọn }\end{array}$ & 5 & 0,5 & 0 & 0 & 0 & 1 & 1 \\
\hline
\end{tabular}




\begin{tabular}{|c|c|c|c|c|c|c|c|c|}
\hline $\begin{array}{l}\text { Quan điểm } \\
\text { nghiên cứu }\end{array}$ & $\begin{array}{c}\text { Miêu tả quan điểm và mối liên } \\
\text { quan đến đánh giá }\end{array}$ & 6 & 1 & 1 & 1 & 1 & 1 & 1 \\
\hline $\begin{array}{l}\text { Đối tượng } \\
\text { so sánh }\end{array}$ & $\begin{array}{l}\text { Mô tả các can thiêp và lý do } \\
\text { lựa chọn can thiệp/chiến lược }\end{array}$ & 7 & 1 & 1 & 1 & 1 & 1 & 1 \\
\hline $\begin{array}{c}\text { Khung thời } \\
\text { gian }\end{array}$ & $\begin{array}{c}\text { Có khung thời gian theo dõi rõ } \\
\text { ràng }\end{array}$ & 8 & 1 & 1 & 1 & 1 & 1 & 1 \\
\hline $\begin{array}{l}\text { Tỷ lề chiết } \\
\text { khấu }\end{array}$ & $\begin{array}{c}\text { Có cho chi phí vào và thông sô } \\
\text { đâu ra, có lý do chọn }\end{array}$ & 9 & 1 & 1 & 1 & 1 & 1 & 0.5 \\
\hline $\begin{array}{l}\text { Lựa chọn yếu } \\
\text { tố đầu ra }\end{array}$ & $\begin{array}{c}\text { Miêu tả thông số đâu ra và } \\
\text { ảnh hưởng }\end{array}$ & 10 & 1 & 1 & 1 & 1 & 1 & 1 \\
\hline \multirow{2}{*}{$\begin{array}{l}\text { Đo lường } \\
\text { chi phí }\end{array}$} & \begin{tabular}{|c|} 
Nghiên cứu đơn: Miêu tả đặc \\
điểm thiết kế nghiên cứu
\end{tabular} & $11 \mathrm{~A}$ & NA & NA & NA & NA & NA & NA \\
\hline & $\begin{array}{l}\text { Tống hợp: Phương pháp chọn } \\
\text { và tổng hợp dữ liệu }\end{array}$ & $11 \mathrm{~B}$ & 0,5 & 1 & 0,5 & 1 & 1 & 0.5 \\
\hline $\begin{array}{l}\text { Yếu tố đầu } \\
\text { ra ưu tiên }\end{array}$ & $\begin{array}{c}\text { Có phương pháp đế gợi ý các } \\
\text { kêt quả ưu tiên }\end{array}$ & 12 & 1 & 1 & 1 & 1 & 1 & 1 \\
\hline \multirow{2}{*}{\begin{tabular}{|c|} 
Uớc tính chi \\
phí nguồn \\
lực
\end{tabular}} & $\begin{array}{c}\text { Nghiên cứu đơn: Phương pháp } \\
\text { đánh giá nguồn lực }\end{array}$ & $13 \mathrm{~A}$ & NA & NA & NA & NA & NA & NA \\
\hline & $\begin{array}{l}\text { Dựa trên mô hình: Cách tiếp } \\
\text { cận và nguồn dự liệu }\end{array}$ & $13 B$ & 1 & 1 & 1 & 1 & 1 & 1 \\
\hline Tỷ giá & $\begin{array}{c}\text { Báo cáo thời gian đánh giá số } \\
\text { lượng và chi phí đơn vị }\end{array}$ & 14 & 1 & 1 & 1 & 1 & 1 & 1 \\
\hline $\begin{array}{l}\text { Lựa chọn } \\
\text { mô hình }\end{array}$ & $\begin{array}{l}\text { Miêu tá và đưa ra lý do lựa } \\
\text { chọn loại mô hình }\end{array}$ & 15 & 1 & 1 & 1 & 1 & 1 & 1 \\
\hline \begin{tabular}{|l|l} 
Giả thiết & 1 \\
\end{tabular} & $\begin{array}{c}\text { Miêu tả toàn bộ cấu trúc hoặc } \\
\text { những giả thiết }\end{array}$ & 16 & 1 & 1 & 0,5 & 1 & 1 & 1 \\
\hline $\begin{array}{c}\text { Phương } \\
\text { pháp phân } \\
\text { tích }\end{array}$ & $\begin{array}{l}\text { Miêu tả tất các phương pháp } \\
\text { phân tích sử dụng }\end{array}$ & 17 & 0,5 & 1 & 1 & 1 & 1 & 1 \\
\hline \multicolumn{9}{|c|}{ Kêtt quả } \\
\hline \begin{tabular}{|c|} 
Biến số \\
nghiên cứu
\end{tabular} & $\begin{array}{c}\text { Có giá trị, khoảng giá trị, xác } \\
\text { suất phân bố các biến }\end{array}$ & 18 & 1 & 1 & 1 & 1 & 1 & 1 \\
\hline $\begin{array}{c}\text { Chi phí và } \\
\text { đâu ra tăng } \\
\text { thêm }\end{array}$ & $\begin{array}{c}\text { Trung bình đâu vào, đầu ra, } \\
\text { ICER nếu có }\end{array}$ & 19 & 1 & 1 & 1 & 1 & 1 & 1 \\
\hline \multirow{2}{*}{$\begin{array}{c}\text { Đặc điểm } \\
\text { của tham sôf }\end{array}$} & $\begin{array}{l}\text { Nghiên cứu đơn: Có ảnh } \\
\text { hưởng của tính bất định }\end{array}$ & $20 \mathrm{~A}$ & NA & NA & NA & NA & NA & NA \\
\hline & $\begin{array}{c}\text { Dựa trên mô hình: Kết quả } \\
\text { phân tích tính bất định }\end{array}$ & $20 B$ & 1 & 1 & 1 & 1 & 1 & 1 \\
\hline $\begin{array}{l}\text { Đặc trưng } \\
\text { của dị biệt }\end{array}$ & $\begin{array}{c}\text { Giải thích thông tin khác biệt } \\
\text { giữa các nhóm }\end{array}$ & 21 & 0,5 & 0,5 & 0,5 & 1 & 0,5 & 1 \\
\hline \multicolumn{9}{|c|}{ Bàn luận } \\
\hline $\begin{array}{l}\text { Kết quả, giới } \\
\text { hạn, tống } \\
\text { quát hóa, } \\
\text { kiến thức } \\
\text { hiện tại } \\
\end{array}$ & $\begin{array}{l}\text { Uu điểm, hạn chế, tính ứng } \\
\text { dụng, sự phù hợp kiến thức } \\
\text { hiện tại }\end{array}$ & 22 & 1 & 1 & 1 & 1 & 1 & 1 \\
\hline \multicolumn{9}{|c|}{ Khác } \\
\hline $\begin{array}{c}\text { Nguî̀n tài } \\
\text { trợ }\end{array}$ & $\begin{array}{c}\text { Báo cáo nguồn tài trợ, ảnh } \\
\text { hưởng nếu có }\end{array}$ & 23 & $\mathrm{NA}$ & NA & NA & NA & NA & NA \\
\hline \multirow[t]{2}{*}{\begin{tabular}{|c|} 
Bất đồng \\
quan điểm \\
\end{tabular}} & $\begin{array}{l}\text { Miêu tả bất kỳ bất đồng nào } \\
\text { giữa các tác giả }\end{array}$ & 24 & 1 & 1 & 1 & 1 & 1 & 1 \\
\hline & Tống điểm & 24 & 21 & 21,5 & 20,5 & 22 & 22,5 & 22 \\
\hline
\end{tabular}

Ghi chú: 0: Không; 1: Có; 0,5: Một phần; NA: No Available (Không có thông tin được ghi nhận); 
Đánh giá chất lượng của các kết quả nghiên cứu dựa vào công cụ CHEERS được thể hiện trong Bảng 1. Các nghiên cứu được đánh giá bằng 24 tiêu chí của công cụ CHEERS: điểm trung bình đạt 21,6/24 điểm và $100 \%$ các nghiên cứu đưa vào đánh giá đạt mức >19/24 điểm.

\subsection{Kết quả các nghiên cứu}

Bảng 2. Tóm tắt các kêt quả nghiên cứu

\begin{tabular}{|c|c|c|c|c|c|c|c|c|c|c|}
\hline \begin{tabular}{|l} 
Tác giả \\
(năm \\
xuất bản) \\
- Năm \\
quy đổi
\end{tabular} & $\begin{array}{l}\text { Quốc } \\
\text { gia- } \\
\text { Quan } \\
\text { điểm }\end{array}$ & $\begin{array}{c}\text { Loại } \\
\text { mồ } \\
\text { hình - } \\
\text { Tài trợ }\end{array}$ & $\begin{array}{l}\text { Thời } \\
\text { gian }\end{array}$ & $\begin{array}{l}\text { Chiết } \\
\text { khấu }\end{array}$ & $\begin{array}{l}\text { Diêu } \\
\text { trị }\end{array}$ & $\begin{array}{l}\text { Đối } \\
\text { tượng }\end{array}$ & $\begin{array}{l}\text { Can thiệp } \\
\text { so sánh }\end{array}$ & $\begin{array}{l}\text { Ngưỡng } \\
\text { chi trả }\end{array}$ & Kết quả & $\begin{array}{l}\text { Kết } \\
\text { luận }\end{array}$ \\
\hline & & & & & & & \multicolumn{2}{|c|}{$\begin{array}{c}\text { Erlotinib so với } \\
\text { hóa trị }\end{array}$} & & \\
\hline $\begin{array}{l}\text { Alain và } \\
\text { cộng sự } \\
\text { (CS) [1] } \\
\text { (2016) - } \\
2015 \\
\end{array}$ & $\begin{array}{c}y ́ \text { - Người } \\
\text { bệnh chi } \\
\text { trả } \\
\text { (NBCT) }\end{array}$ & $\begin{array}{l}\text { Markov } \\
\text { - Không }\end{array}$ & $\begin{array}{c}4 \\
\text { năm }\end{array}$ & $3 \%$ & $\begin{array}{l}\text { Bước } \\
\text { một }\end{array}$ & \begin{tabular}{|l} 
Giai \\
đoạn \\
tiển \\
triển
\end{tabular} & $\begin{array}{l}\text { Erlotinib so } \\
\text { với CDGC }\end{array}$ & $\begin{array}{l}\text { 76.051,23 } \\
\$ / Q A L Y *\end{array}$ & $\begin{array}{c}\text { CP tiết kiệm: } \\
\text { 18.474,92 \$* } \\
\text { QALY gia tăng: } \\
0,117 .\end{array}$ & $\begin{array}{l}\text { Erlotinib } \\
\text { có CP } \\
\text { tiết kiệm } \\
\text { hơn. }\end{array}$ \\
\hline $\begin{array}{l}\text { Alain và } \\
\text { cộng sự } \\
\text { (CS)[1] } \\
(2016)- \\
2015\end{array}$ & $\begin{array}{c}\text { Tây Ban } \\
\text { Nha - } \\
\text { NBCT }\end{array}$ & $\begin{array}{l}\text { Markov } \\
\text { - Không }\end{array}$ & $\begin{array}{c}4 \\
\text { năm }\end{array}$ & $3 \%$ & $\begin{array}{l}\text { Bước } \\
\text { một }\end{array}$ & $\begin{array}{l}\text { Giai } \\
\text { đoạn } \\
\text { tiển } \\
\text { triển }\end{array}$ & $\begin{array}{l}\text { Erlotinib so } \\
\text { với CDGC }\end{array}$ & $\begin{array}{l}\text { 96.051,23 } \\
\$ / \text { QALY* }^{*}\end{array}$ & $\begin{array}{c}\text { CP tiết kiêem: } \\
8.331,91 \text { \$*, } \\
\text { QALY gia tăng: } \\
0,117 .\end{array}$ & $\begin{array}{l}\text { Erlotinib } \\
\text { có CP } \\
\text { tiết kiệm } \\
\text { hơn. }\end{array}$ \\
\hline $\begin{array}{l}\text { Alain và CS } \\
{[1](2016)} \\
-2015\end{array}$ & $\begin{array}{l}\text { Pháp - } \\
\text { NBCT }\end{array}$ & $\begin{array}{c}\text { Markov } \\
\text { - Không }\end{array}$ & $\begin{array}{c}4 \\
\text { năm }\end{array}$ & $3 \%$ & $\begin{array}{l}\text { Bước } \\
\text { một }\end{array}$ & \begin{tabular}{|c|} 
Giai \\
đoạn \\
tiển \\
triển \\
\end{tabular} & $\begin{array}{l}\text { Erlotinib so } \\
\text { với CDGC }\end{array}$ & $\begin{array}{l}\text { 76.051,23 } \\
\$ / Q A L Y *\end{array}$ & \begin{tabular}{|c|} 
CP tiết kiệm: \\
20.665,96 \$* \\
QALY gia tăng: \\
$0,117$. \\
\end{tabular} & $\begin{array}{l}\text { Erlotinib } \\
\text { có CP } \\
\text { tiết kiệm } \\
\text { hơn. }\end{array}$ \\
\hline \multirow[t]{2}{*}{\begin{tabular}{|c|} 
Siying \\
Wang và \\
CS \\
[2] (2013) \\
-2010 \\
\end{tabular}} & $\begin{array}{c}\text { Trung } \\
\text { Quốc- } \\
\text { HTCSSK }\end{array}$ & $\begin{array}{c}\text { Markov } \\
\text { - Không }\end{array}$ & $\begin{array}{c}5 \\
\text { năm }\end{array}$ & $3 \%$ & $\begin{array}{l}\text { Bước } \\
\text { một }\end{array}$ & \begin{tabular}{|l} 
Giai \\
đoạn \\
tiến \\
triển
\end{tabular} & $\begin{array}{l}\text { Erlotinib so } \\
\text { với CG }\end{array}$ & $\begin{array}{l}96.884 \\
\$ / Q A L Y\end{array}$ & $\begin{array}{c}\text { ICER: } 85.927,4 \\
\text { \$/QALY. }\end{array}$ & $\begin{array}{l}\text { Erlotinib } \\
\text { có CPHQ } \\
\text { hơn. }\end{array}$ \\
\hline & & & & & & & \multicolumn{2}{|c|}{$\begin{array}{c}\text { Afatinib so với hóa } \\
\text { trị }\end{array}$} & & \\
\hline \begin{tabular}{|c|} 
Xiaohua Gu \\
và CS [3] \\
(2019) - \\
2016 \\
\end{tabular} & $\begin{array}{l}\text { Trung } \\
\text { Quốc- } \\
\text { HTCSSK }\end{array}$ & $\begin{array}{l}\text { Markov } \\
\text { - Không }\end{array}$ & $\begin{array}{c}10 \\
\text { năm }\end{array}$ & $5 \%$ & $\begin{array}{l}\text { Bước } \\
\text { một }\end{array}$ & \begin{tabular}{|l|} 
Giai \\
đoạn \\
tiển \\
triển \\
\end{tabular} & $\begin{array}{c}\text { Afatinib với } \\
\text { PC }\end{array}$ & $\begin{array}{l}23.815 \\
\text { \$/QALY }\end{array}$ & $\begin{array}{l}\text { ICER: } 20.758 \\
\text { \$/QALY. }\end{array}$ & $\begin{array}{l}\text { Afatinib } \\
\text { có CPHQ } \\
\text { hơn. }\end{array}$ \\
\hline \multirow[t]{2}{*}{$\begin{array}{c}\text { Ruxu You } \\
\text { và CS [4] } \\
(2019)- \\
2019\end{array}$} & $\begin{array}{l}\text { Trung } \\
\text { Quốc- } \\
\text { HTCSSK }\end{array}$ & \begin{tabular}{|c|} 
Cây \\
quyết \\
đinh - \\
Không
\end{tabular} & $\begin{array}{c}5 \\
\text { năm } \\
\text { trọn } \\
\text { đời }\end{array}$ & $5 \%$ & $\begin{array}{l}\text { Bước } \\
\text { một }\end{array}$ & $\begin{array}{l}\text { Giai } \\
\text { đoạn } \\
\text { tiển } \\
\text { triển }\end{array}$ & $\begin{array}{l}\text { Afatinib so } \\
\text { với GC }\end{array}$ & $\begin{array}{l}26.508 \\
\$ / Q A L Y\end{array}$ & $\begin{array}{c}\text { ICER là } \\
\text { 33.416,39 } \\
\text { \$/QALY. Theo } \\
\text { hương trình hỗ } \\
\text { trợ bệnh nhân, } \\
\text { ICER: 22.972,5 } \\
\text { USD/QALY. } \\
\end{array}$ & $\begin{array}{l}\text { Afatinib } \\
\text { có CPHQ } \\
\text { hơn. }\end{array}$ \\
\hline & & & & & & & \multicolumn{2}{|c|}{$\begin{array}{c}\text { Osimertinib so với } \\
\text { hóa trị }\end{array}$} & & \\
\hline \begin{tabular}{|c|} 
Evelina \\
Bertranou \\
và CS [5] \\
$(2017)-$ \\
2017 \\
\end{tabular} & $\begin{array}{l}\text { Anh - } \\
\text { NBCT }\end{array}$ & $\begin{array}{l}\text { Cây } \\
\text { quyết } \\
\text { định - } \\
\text { Không }\end{array}$ & $\begin{array}{c}15 \\
\text { năm }\end{array}$ & $3,5 \%$ & $\begin{array}{l}\text { Bước } \\
\text { sau }\end{array}$ & $\begin{array}{l}\text { Sau } \\
\text { thất } \\
\text { bại } \\
\text { pước } 1\end{array}$ & $\begin{array}{l}\text { Psimertinib } \\
\text { với PDC }\end{array}$ & $\begin{array}{l}\text { 61.881,2 } \\
\$ / Q A L Y^{* *}\end{array}$ & $\begin{array}{l}\text { ICER: } \\
51.615,099 \$ \\
\text { /QALY**. }\end{array}$ & $\begin{array}{l}\text { Osimerti } \\
\text { nib có } \\
\text { CPHQ } \\
\text { hon. }\end{array}$ \\
\hline \begin{tabular}{|c|} 
Haijing \\
Guan và CS \\
[6] (2019) \\
-2018 \\
\end{tabular} & $\begin{array}{l}\text { Trung } \\
\text { Quốc- } \\
\text { HTCSSK }\end{array}$ & $\begin{array}{c}\text { Markov } \\
\text { - Không }\end{array}$ & $\begin{array}{c}10 \\
\text { năm }\end{array}$ & $3 \%$ & $\begin{array}{l}\text { Bước } \\
\text { sau }\end{array}$ & $\begin{array}{l}\text { Sau } \\
\text { thất } \\
\text { baí } \\
\text { pước } 1\end{array}$ & $\begin{array}{l}\text { Osimertinib } \\
\text { vói PP, GP, } \\
\text { DP, TP }\end{array}$ & \begin{tabular}{|}
$30.000-$ \\
50.000 \\
$\$ / Q A L Y$ \\
\end{tabular} & $\begin{array}{c}\text { ICER: } 23.760,1 \\
-23.994,1 \\
\text { \$/QALY. }\end{array}$ & $\begin{array}{c}\text { Osimerti } \\
\text { nib có } \\
\text { CPHQ } \\
\text { hon. }\end{array}$ \\
\hline
\end{tabular}


Ghi chú: HTCSSK: Hệ thống chăm sóc sức khỏe Trung Quốc; CDGC: cisplatin, docetaxel, gemcitabine, carboplatin; CG: carboplatingemcitabine; PC: pemetrexed - cisplatin; GC: gemcitabine-cisplatin; PDC: pemetrexed + carboplatin hoặc cisplatin; 4 liệu pháp hóa trị: pemetrexed + platinum (PP), gemcitabine + platinum (GP), docetaxel + platinum (DP) ), và paclitaxel + platinum (TP).

*Tỷ giá chuyển đổi giữa \$ và $€$ (năm 2015); **Tỷ giá chuyển đổi giữa \$ và $£$ (năm 2017);

Mỗi nghiên cứu trong 6 kết quả được lựa chọn sẽ có những hạn chế riêng: phương pháp đánh giá, thời gian, nguồn tài trợ, quốc gia, kết hợp điều trị và phương pháp điều trị,... Tuy nhiên, các kết quả đều thể hiện đầy đủ các thông tin cần quan tâm: ICER, QALY,.. (Bảng 2).

\section{BÀN LUÂ̂N}

Erlotinib so với hóa trịCác nghiên cứu đều chỉ ra erlotinib có $\mathrm{CPHQ}$ hơn so với hóa trị liệu trong điều trị đầu tay ở bệnh nhân UTPKTBN [12]. Erlotinib mang lại QALY: 0.117 so với hóa trị và tiết kiệm $\mathrm{CP}$ lần lượt là $8.331,91$ * (Tây Ban Nha); 18.474,92 \$* (Ý); 20.665,96 \$* (Pháp) [1]. Mặt khác, theo nghiên cứu tại 22 trung tâm ở Trung Quốc, Erlotinib có QALY gia tăng là 0,56 năm và ICER dao động từ \$58.584,57 đến \$ $336.404,20$ ở ngưỡng chi trả 96.884 đô la so với hóa trị liệu cơ bản CDGC [1]. Theo nghiên cứu của Siying Wang tại Trung Quốc thì erlotinib có chi phí hiệu quả so với carboplatin-gemcitabine với ICER: 85.927,4 \$/QALY [2]. Như vậy, các nghiên cứu đều có cùng quan điểm chung là Erlotinib có CPHQ hơn so với hóa trị liệu.

Afatinib so với hóa trị. Các nghiên cứu đều chỉ ra afatinib là liệu pháp điều trị hiệu quả về chi phí so với hóa trị liệu trong điều trị đầu tay của UTPKTBN [3-4]. Afatinib đã gia tăng ICER: 20.758 \$/QALY so với hóa trị (pemetrexed cisplatin) [3]. Theo chương trình hỗ trợ bệnh nhân (PAP), ICER của afatinib đạt 22.972,5 $\$ / Q A L Y$ và có $C P H Q$ so với gemcitabine-cisplatin [4]. Việc giảm giá afatinib từ $10 \%$ đến $30 \%$ đã làm giảm ICER xuống còn 24.562,93 - 30.714,93 \$/QALY [4]. Do vậy, năm 2014 afatinib được khuyến nghị như một lựa chọn đầu tay để điểu trị cho người bệnh mắc bệnh UTPKTBN dương tính với đột biến gen EGFR-TK [4].

Osimertinib so với hóa trị. Các nghiên cứu đều chỉ ra Osimertinib có CPHQ cao hơn so với hóa trị liệu trong điều trị bước sau của UTPKTBN dương tính với EGFR [5-6]. Osimertinib có ICER đạt từ 23.760,1 - 23.994,1 \$/QALY so với pemetrexed + platinum (PP), gemcitabine + platinum (GP), docetaxel + platinum (DP) ), và paclitaxel + platinum (TP) [6]. Tương tự, osimertinib đạt được ICER: 51.615,099\$ /QALY so với PDC với ngưỡng chi trả là $61.881,2$ \$/QALY** [5]. Do vậy, Osimertinib là lựa chọn điều trị cho bước sau có $\mathrm{CPHQ}$ cao hơn so với các liệu pháp hóa trị.

\section{KẾT LUẬN VÀ KHUYẾN NGH!}

Erlotinib và afatinib là thuốc điều trị bước một và osimertinib là thuốc điêu trị bước sau có CPHQ tốt hơn so với hóa trị liệu. Giảm giá thuốc điều trị nhắm trúng đích thấp hơn so với ngưỡng chi trả của từng nước là yếu tố thuận lợi để người bệnh UTPKTBN có khả năng tiếp cận và gia tăng hiệu quả về chi phí.

\section{TÀI LIỆU THAM KHẢO}

1. Vergnenegre A, Massuti $B$, de Marinis $F$, et al (2016). Spanish Lung Cancer Group, Italian Association of Thoracic Oncology, and French Lung Cancer Group. Economic Analysis of First-Line Treatment with Erlotinib in an EGFR-Mutated Population with Advanced NSCLC. J Thorac Oncol, 11(6), 801-807.

2. Wang S, Peng L, Li J, et al (2018). A trial-based cost-effectiveness analysis of erlotinib alone versus platinum-based doublet chemotherapy as first-line therapy for Eastern Asian nonsquamous non-smallcell lung cancer. PLoS One, 8(3), e55917.

3. Gu X, Zhang Q, Chu YB, et al (2018). Costeffectiveness of afatinib, gefitinib, erlotinib and pemetrexed-based chemotherapy as first-line treatments for advanced non-small cell lung cancer in China. Lung Cancer, 127, 84-89.

4. You $R$, Liu J, Wu DB, et al (2019). CostEffectiveness Analysis Of EGFR Mutation Testing And Afatinib Versus Gemcitabine-Cisplatin As FirstLine Therapy For Advanced Non-Small-Cell Lung Cancer In China. Cancer Manag Res, 11, 10239-10248.

5. Bertranou E, Bodnar C, Dansk V, et al (2019). Cost-effectiveness of osimertinib in the UK for advanced EGFR-T790M non-small cell lung cancer. J Med Econ; 21(2), 113-121.

6. Guan H, Liu G, Xie F, et al (2019). Costeffectiveness of Osimertinib as a Second-line Treatment in Patients With EGFR-mutated Advanced Non-Small Cell Lung Cancer in China. Clin Ther, 41(11), 2308-2320.

7. Organisation for Economic Co-Operation and Development (OECD): StatExtracts: PPPs and Exchange

Rates. http://stats.oecd.org/Index.aspx?DatasetCo de=SNA_TABLE 4.

8. Husereau, Drummond, Petrou, et al (2013). Consolidated Health Economic Evaluation Reporting Standards (CHEERS) Statement. BMJ 2013, 346, f1049-f1049. 